\title{
Myeloid-related protein-8/14 facilitates bacterial growth during pneumococcal pneumonia
}

\author{
Ahmed Achouiti, ${ }^{1}, 2$ Thomas Vogl, ${ }^{3}$ Henrik Endeman, ${ }^{4}$ Brittany L Mortensen, ${ }^{5}$ \\ Pierre-Francois Laterre, ${ }^{6}$ Xavier Wittebole, ${ }^{6}$ Marieke A D van Zoelen, ${ }^{7}$ \\ Yaofang Zhang, ${ }^{5}$ Jacobien J Hoogerwerf, ${ }^{1,2}$ Sandrine Florquin, ${ }^{8}$ Marcus J Schultz, ${ }^{9,10}$ \\ Jan C Grutters, ${ }^{11,12}$ Douwe H Biesma, ${ }^{13}$ Johannes Roth, ${ }^{3}$ Eric P Skaar, ${ }^{5}$ Cornelis van \\ 't Veer, ${ }^{1,2}$ Alex F de Vos, ${ }^{1,2}$ Tom van der Poll ${ }^{1,2,14}$
}

- Additional material is published online only. To view please visit the journal online (http://dx.doi.org/10.1136/ thoraxjnl-2014-205668)

For numbered affiliations see end of article.

\section{Correspondence to} Ahmed Achouiti, Academic Medical Center, Center for Experimental and Molecular Medicine, Meibergdreef 9 , Room G2-130, Amsterdam 1105 AZ, The Netherlands; a.achouiti@amc.uva.nl

Received 6 May 2014 Revised 7 August 2014 Accepted 8 August 2014 Published Online First 1 September 2014
CrossMark

To cite: Achouiti A, Vogl T, Endeman $\mathrm{H}$, et al. Thorax 2014;69:1034-1042.

\section{ABSTRACT}

Background Streptococcus pneumoniae is the most commonly identified pathogen in community-acquired pneumonia (CAP). Myeloid-related protein (MRP) $8 / 14$ is a major component of neutrophils that is released upon infection or injury. MRP8/14 is essential for protective immunity during infection by a variety of microorganisms through its capacity to chelate manganese and zinc. Here, we aimed to determine the role of MRP8/14 in pneumococcal pneumonia.

Methods MRP8/14 was determined in bronchoalveolar lavage fluid (BALF) and serum of CAP patients, in lung tissue of patients who had succumbed to pneumococcal pneumonia, and in BALF of healthy subjects challenged with lipoteichoic acid (a component of the gram-positive bacterial cell wall) via the airways. Pneumonia was induced in MRP14 deficient and normal wildtype mice. The effect of MRP8/14 on S. pneumoniae growth was studied in vitro.

Results CAP patients displayed high MRP8/14 levels in BALF, lung tissue and serum. Healthy subjects challenged with lipoteichoic acid demonstrated elevated MRP8/14 in BALF. Likewise, mice with pneumococcal pneumonia had high MRP8/14 levels in lungs and the circulation. MRP14 deficiency, however, was associated with reduced bacterial growth and lethality, in the absence of notable effects on the inflammatory response. High zinc levels strongly inhibited growth of S. pneumoniae in vitro, which was partially reversed by MRP8/14.

Conclusions In sharp contrast to its previously reported host-protective role in several infections, the present results reveal that in a model of CAP, MRP8/14 is misused by S. pneumoniae, facilitating bacterial growth by attenuating zinc toxicity toward the pathogen.

\section{INTRODUCTION}

Community-acquired pneumonia (CAP) is a common infectious disease and a leading cause of sepsis. ${ }^{1} 2$ The most prevalent causative microorganism in CAP is the Gram-positive diplococcus Streptococcus pneumoniae. ${ }^{1-3}$ Pneumococci are increasingly antimicrobial resistant, ${ }^{4}$ thus stressing the importance of gaining more insight into host defence mechanisms that influence the outcome of this prominent infection.

\section{Key messages}

What is the key question?

- What is the role of myeloid-related protein (MRP)8/14 in Streptococcus pneumoniaeinduced community-acquired pneumonia (CAP) in a clinical and experimental setting?

\section{What is the bottom line?}

- MRP8/14 is compartmentally released in CAP and is associated with increased lethality in a mouse model of CAP through the facilitation of pneumococcal growth by attenuating zinc toxicity toward the pathogen.

\section{Why read on?}

- In sharp contrast to the previously reported host protective role of MRP8/14 in several infections, this study represents the first in vivo example of how a pathogen can misuse this protein for its own benefit.

When pneumococci invade the lower respiratory tract, various inflammatory mechanisms ensue, including infiltration and activation of neutrophils, ${ }^{35}$ which is associated with the release of complexes of myeloid-related protein (MRP) 8 and MRP14 (MRP8/14 or calprotectin). ${ }^{6} \mathrm{MRP} 8 / 14$ induces a variety of innate immune responses, including induction of leukocyte recruitment and cytokine release via Toll-like receptor (TLR) $4 .^{7}$ In addition to its proinflammatory effects, MRP8/14 possesses direct antimicrobial properties directed against bacteria $^{8-10}$ and fungi. ${ }^{11} 12$ MRP8/14 mediates at least part of these effects by chelation of manganese and zinc, elements that are generally important for microbial growth and virulence. ${ }^{13}$ Previous investigations have shown that manganese is critical for pneumococci to manage oxidative stress, allowing growth under normal aerobic conditions. ${ }^{14-17}$ Importantly, however, while low amounts of zinc are required for growth of S. pneumoniae as well, ${ }^{18}$ high zinc concentrations are toxic for this micro-organism due to competition with manganese for the binding to pneumococcal surface adhesin A (PsaA), the solute-binding 
protein of a specific manganese transporter, thereby preventing manganese uptake. ${ }^{14-17}$

Our group previously reported elevated plasma MRP8/14 levels in patients with severe pneumonia. ${ }^{19}$ Here, we speculated that MRP8/14 is released at sites of infection, and that in pneumonia caused by $S$. pneumoniae, MRP8/14 may significantly impact on host defence, at least in part, by interfering with the availability of zinc. Therefore, we set out to determine the role of MRP8/14 in CAP and pneumococcal pneumonia by combining observational studies in humans with functional studies in mice.

\section{METHODS}

\section{Human studies}

Two groups of consecutive CAP patients were studied. ${ }^{1}$ Eight patients admitted to the intensive care unit (ICU) of St Luc University Hospital (Leuven, Belgium), from whom bronchoalveolar lavage fluid (BALF) was obtained from the site of infection as visualised on the chest X-ray ${ }^{2} ; 7$ patients admitted to the emergency department (ED) of St Antonius Hospital (Nieuwegein, The Netherlands), from whom BALF was obtained from both the infected and contralateral uninfected lung, as well as serum on admission and 1,2, 5, 10 and 30 days thereafter. Twenty-four patients without lung disease scheduled to undergo elective surgery in whom BALF was collected directly after induction of anaesthesia served as a control. ${ }^{20}$ Additionally, we analysed BALF samples from a previous study $^{21}$ in which eight healthy subjects (mean age 23.4 \pm 1.3 years) who were challenged with $100 \mathrm{ng} / \mathrm{kg}$ lipoteichoic acid (LTA), from Staphylococcus aureus (Deutsche Sammlung von Mikroorganismen 20233) ${ }^{22}$ into a lung subsegment and sterile saline into the contralateral lung. ${ }^{21}$ Bilateral BAL was performed $6 \mathrm{~h}$ thereafter. The institutional ethics committees approved all human studies and written informed consent was obtained from all patients or their relatives. MRP8/14 was measured by ELISA. ${ }^{23}$ Staining of MRP8 and MRP14 of archival postmortem lung tissue from patients who had died from pneumococcal pneumonia $(n=3)$ or from a non-pulmonary cause $(n=3)$ was done as described. ${ }^{24}$

\section{Mouse studies}

The Animal Care and Use Committee of the University of Amsterdam approved all experiments. $\mathrm{C} 57 \mathrm{Bl} / 6$ wildtype (wt) mice (Charles River Laboratories, Maastricht, The Netherlands) and MRP14 deficient $\left(\mathrm{Mrp14^{-/ }}\right)$ mice $^{24}{ }^{25}$ (backcrossed >10 times to a $\mathrm{C} 57 \mathrm{Bl} / 6$-background) were intranasally inoculated with $5 \times 10^{3}$ or $5 \times 10^{4}$ colony forming units (cfu) S. pneumoniae serotype 3 (ATCC 6303 Rockville, Maryland, USA) ( $\mathrm{n}=7$ -8 per strain) and sacrificed 6,24 or 48 h thereafter. Collection and handling of samples, and quantitative cultures were done as described. ${ }^{26} 27$ In survival studies, mice $(n=12-16$ per strain) were monitored for up to 7 days after infection $\left(5 \times 10^{4} \mathrm{cfu}\right)$. BALF was obtained from a separate group of infected wt mice $(n=6)$ at indicated time points as described. ${ }^{26} 27$ See online supplement for cytokine measurements and histology.

\section{In vitro studies}

See online supplement.

\section{Statistical analysis}

Data are expressed as box-and-whisker diagrams unless indicated otherwise. Differences within individual patients or volunteers were analysed by Wilcoxon-matched pairs test. Differences between patients and volunteers, or wt and $m r p 14^{-/-}$mice were analysed by Mann-Whitney U test. Survival was compared by Kaplan-Meier analysis followed by a log rank test. Values of $\mathrm{p}<0.05$ were considered statistically significant.

\section{RESULTS}

\section{Patients with CAP show elevated lung and plasma MRP8/14 levels}

To obtain a first insight into MRP8/14 release during pneumonia, we measured MRP8/14 levels in BALF from eight CAP patients requiring treatment in the ICU, and from seven CAP patients admitted to the ED (table 1). S. pneumoniae was the most commonly isolated pathogen (in total 7 out of 15 cases, or 46.7\%). Patients admitted to the ICU displayed elevated levels of MRP8/14 in BALF compared with controls (figure 1A, $\mathrm{p}<0.001)$. In agreement, patients admitted to the ED displayed a profound compartmentalised increase of MRP8/14 at the site of infection (figure $1 \mathrm{~B}, \mathrm{p}<0.001$ vs the contralateral lung); these patients also showed raised serum MRP8/14 levels, which remained elevated until 30 days after admission compared with normal serum values (figure $1 \mathrm{C}, \mathrm{p}<0.05$; at other time points: $\mathrm{p}<0.01$ ). To obtain insight in MRP8/14 expression in lung

Table 1 Demographic and clinical characteristics, and microbiology results of patients with community-acquired pneumonia

\begin{tabular}{|c|c|c|c|}
\hline Patient characteristics & $\begin{array}{l}\text { Controls } \\
\text { unilateral BALF, n=24 }\end{array}$ & $\begin{array}{l}\text { ICU cohort } \\
\text { unilateral BALF, } n=8\end{array}$ & $\begin{array}{l}\text { ED cohort } \\
\text { bilateral BALF, } n=7\end{array}$ \\
\hline Age, years $($ mean $\pm S E)$ & $65.1 \pm 2.0$ & $63.9 \pm 3.7$ & $52.4 \pm 6.3$ \\
\hline Male sex (\%) & $17(71)$ & $2(25)$ & $3(43)$ \\
\hline APACHE-II score (mean \pm SE) & Not determined & $27.7 \pm 1.5$ & $15.3 \pm 4.7$ \\
\hline 28-day mortality (\%) & $1(4)$ & $2(25)$ & $0(0)$ \\
\hline \multicolumn{4}{|l|}{ Microbiology } \\
\hline Streptococcus pneumonia & & 3 & 4 \\
\hline Mycoplasma pneumoniae & & - & 1 \\
\hline Haemophilus influenza & & - & 1 \\
\hline Acinetobacter baumannii & & 1 & - \\
\hline Legionella pneumophila & & 1 & - \\
\hline Pneumocystis jeroveci & & 1 & - \\
\hline No micro-organism identified & & 2 & 1 \\
\hline
\end{tabular}



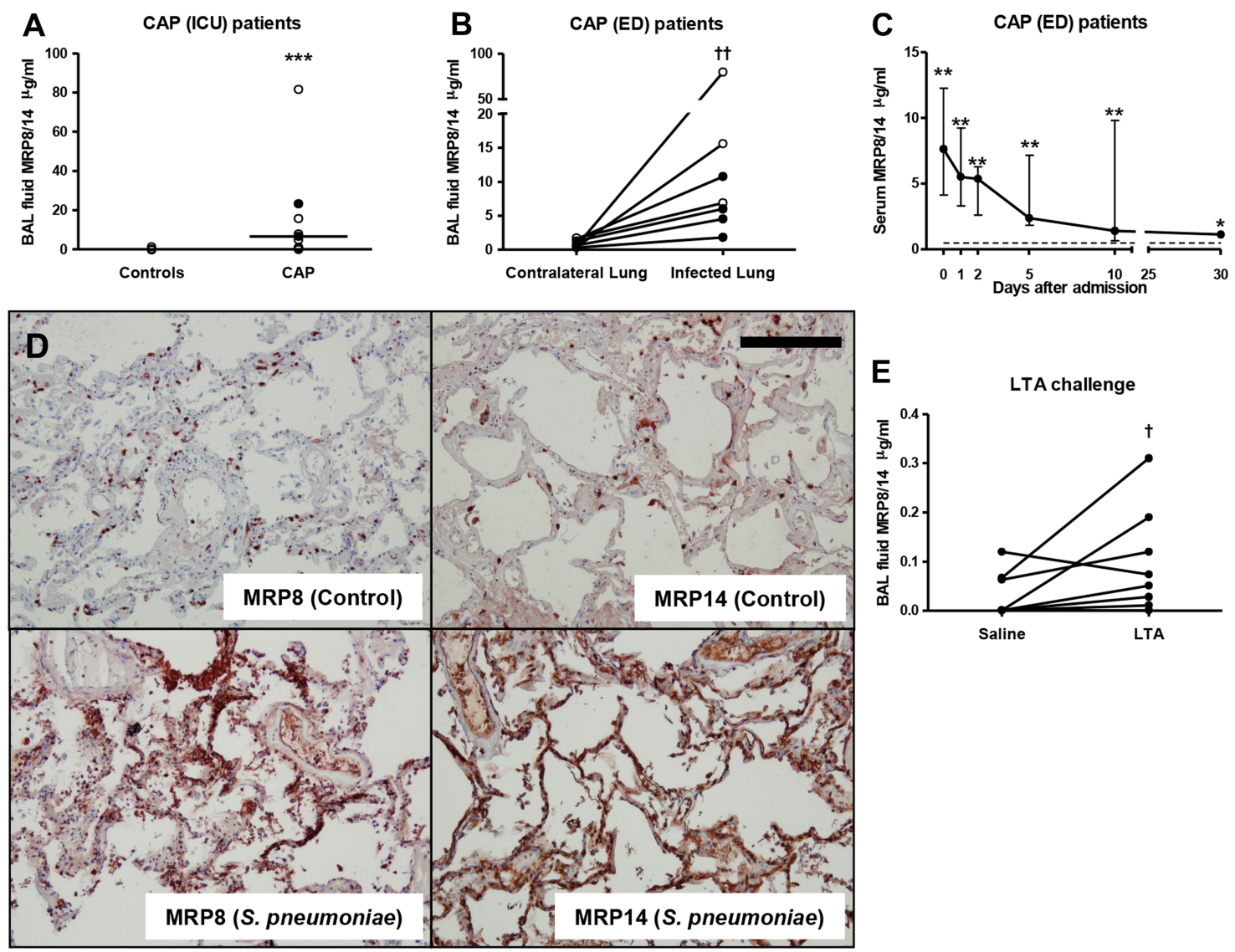

Figure 1 Human community-acquired pneumonia (CAP) and bronchial lipoteichoic acid (LTA) instillation result in an increase of myeloid-related protein (MRP)8/14 in lungs and plasma. (A) MRP8/14 levels in bronchoalveolar lavage fluid (BALF) from control patients and patients admitted to the intensive care unit with CAP. Each dot represents a measurement within an individual patient, with horizontal lines showing medians. Closed dots: Streptococcus pneumoniae pneumonia. Open dots: pneumonia due to other causative pathogen. (B) MRP8/14 levels in BALF from the infected and contralateral non-infected lung from patients admitted to the emergency department (ED) with CAP. Data are expressed as individual measurements (dots). Closed dots: S. pneumoniae pneumonia. Open dots: pneumonia due to other causative pathogen. Connections between dots express measurements from individual patients. (C) Serum MRP8/14 levels in serum from CAP patients admitted to the ED at indicated time points; the broken line represents the normal MRP8/14 value in a healthy individual measured at one timepoint. Data are expressed as median and IQR range. (D) MRP8 and MRP14 stainings in lungs of patients who had died from pneumococcal pneumonia or a non-pulmonary cause. Scalebar indicates $200 \mu \mathrm{m}$. (E) MRP8/14 levels in BALF from healthy subjects $6 \mathrm{~h}$ after intrabronchial instillation of LTA (100 ng/kg) or sterile saline (in the contralateral lung). Data are expressed as individual measurements (dots). Connections between dots express measurements from individual subjects; ${ }^{*} p<0.05,{ }^{* *} p<0.01,{ }^{* *} p<0.001$ versus control BALF or serum, Mann-Whitney $U$ test; ${ }^{\dagger} p<0.05,{ }^{+t} p<0.01$ versus contralateral lung, Wilcoxon matched pairs test.

tissue in human CAP, we performed immunohistochemical staining for MRP8 and MRP14 on stored lung tissue slides of patients who had succumbed to CAP caused by S. pneumoniae and from patients who had died from a non-pulmonary cause. While control lung tissue samples showed a small number of resident cells expressing MRP8 or MRP14, lung tissue from patients who had died from pneumococcal pneumonia demonstrated a strong increase in both MRP8 and MRP14 staining (figure 1D). Together, these data provide strong evidence for local release of MRP8/14 during human CAP at the site of infection.

Next, we determined whether an acute bacterial stimulus can elicit local MRP8/14 release in the bronchoalveolar space of healthy humans. Indeed, $6 \mathrm{~h}$ after bronchial instillation, MRP8/14 concentrations were higher in BALF harvested from the lung segment challenged with LTA (a proinflammatory component of the Gram-positive cell wall) compared with BALF obtained from the saline challenged lung segment $(p<0.05$ vs saline, figure $1 \mathrm{E})$.
Lung and plasma MRP8/14 levels are increased during murine pneumococcal pneumonia

We used our well-established CAP model in mice, produced by intranasal infection with a virulent strain of S. pneumoniae 2627 to obtain insight into the functional role of MRP8/14. After pneumococcal infection $\left(5 \times 10^{4} \mathrm{cfu}\right)$, elevated local levels of MRP8/14 were measured in BALF (median $4.4 \mu \mathrm{g} / \mathrm{mL}$ at $48 \mathrm{~h}$; figure 2A) and whole-lung homogenates (median $77.0 \mu \mathrm{g} / \mathrm{mL}$ at $48 \mathrm{~h}$; figure 2B). In plasma, MRP8/14 was strongly enhanced after $48 \mathrm{~h}$ (median $5.6 \mu \mathrm{g} / \mathrm{mL}$; figure $2 \mathrm{C}$ ). We next stained lung tissue slides from naive and infected mice to establish the cellular source of MRP8 and MRP14 (figure 2D). Naive lungs showed a small number of MRP8 and MRP14 positive cells. MRP8 and MRP14 expressions both strongly increased at later time points, presumably due to large peribronchial neutrophilic infiltrates (see online supplementary figure S1). Together, these data indicate that murine pneumococcal pneumonia resembles human CAP with regard to high pulmonary and systemic expression of MRP8/14. 

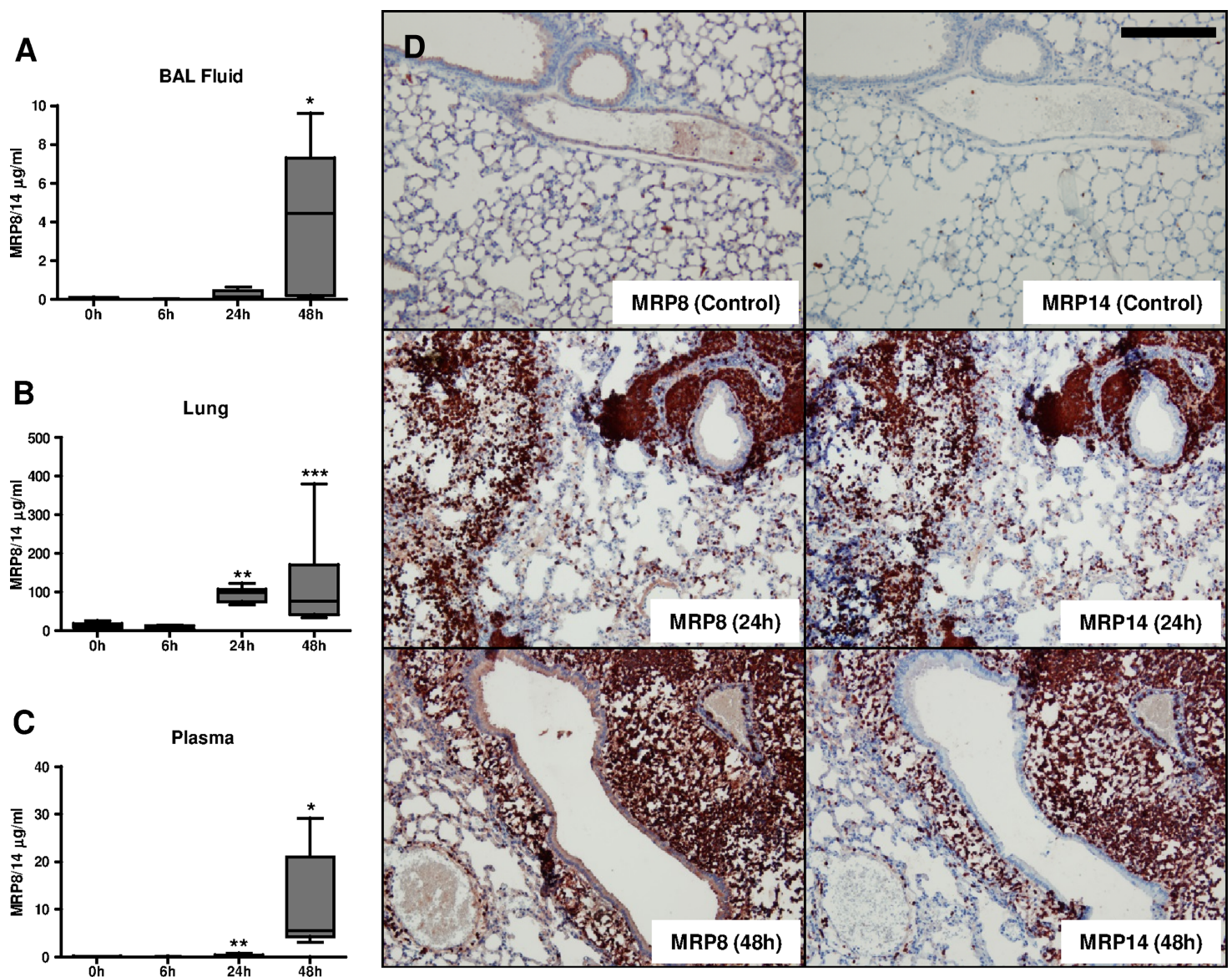

Figure 2 Murine pneumococcal pneumonia results in an increase of myeloid-related protein (MRP)8/14 in lungs and plasma. MRP8/14 levels in bronchoalveolar lavage fluid (A), whole-lung homogenates (B), and plasma (C) in naive mice and 6, 24 and $48 \mathrm{~h}$ after intranasal Streptococcus pneumoniae infection $\left(5 \times 10^{4} \mathrm{cfu}\right)$. Data are expressed as box-and-whisker diagrams depicting the smallest observation, lower quartile, median, upper quartile and largest observation. MRP8 and MRP14 stainings in lungs of naive mice and mice $24 \mathrm{~h}$ or $48 \mathrm{~h}$ after infection with S. pneumoniae (D). Scale bar indicates $200 \mu \mathrm{m} ;{ }^{*} \mathrm{p}<0.05,{ }^{* *} \mathrm{p}<0.01,{ }^{* * *} \mathrm{p}<0.001$ versus uninfected mice.

\section{$\mathrm{Mrp}^{14^{-/}}$mice show delayed mortality and reduced bacterial growth and dissemination during pneumococcal pneumonia}

To investigate a potential role for MRP8/14 in the outcome of pneumococcal pneumonia, we intranasally infected wt and mrp $14^{-/-}$mice with $S$. pneumoniae $\left(5 \times 10^{4} \mathrm{cfu}\right)$ and monitored them for 7 days (figure $3 \mathrm{~A}$ ). Of note, $m r p 14^{-/}$mice are functionally deficient for MRP8/14 as they lack MRP8 on a protein level, probably due to a higher turnover of isolated MRP8 in the absence of its binding partner MRP14. ${ }^{24} 25$ Remarkably, lack of MRP8/14 significantly prolonged survival: median survival times were 2.5 days for wt mice versus 3.7 days for mrp $14^{-/-}$mice $(\mathrm{p}=0.005)$.

We wondered whether the delayed lethality of $\operatorname{mrp} 14^{-/-}$mice was associated with a difference in bacterial growth and dissemination. Therefore, we euthanased wt and $\operatorname{mrp} 14^{-/-}$mice at 6 , 24 or $48 \mathrm{~h}$ after pneumococcal infection $\left(5 \times 10^{4} \mathrm{cfu}\right)$, and examined bacterial loads in lungs, blood, spleens and livers (figure 3B-E). At $6 \mathrm{~h}$, bacterial loads in the lungs of $m r p 14^{-/-}$ mice were similar to those in wt mice. Twenty-four hours after infection, mrp $14^{-/-}$mice displayed reduced bacterial outgrowth in all organs and blood ( $<<0.05$ vs wt mice). After $48 \mathrm{~h}$, $m r p 14^{-/-}$mice showed no difference in pulmonary bacterial loads compared to wt mice, but still lower pneumococcal burdens in blood and spleen $(\mathrm{p}<0.05)$. Similar results were obtained with a 10-fold lower dose of pneumococci $\left(5 \times 10^{3} \mathrm{cfu}\right): 48 \mathrm{~h}$ after infection, mrp $14^{-/-}$mice had similar bacterial loads in their lungs and lower bacterial burdens in blood and distant organs relative to wt mice $(\mathrm{p}<0.01)$. Together, these data indicate that MRP8/14 facilitates bacterial growth in lungs and especially distant organs, thereby contributing to accelerated lethality during pneumococcal pneumoniaderived sepsis.

\section{MRP14 deficiency has little impact on lung inflammation}

To evaluate the role of MRP14 in lung inflammation we analysed lung tissue slides obtained from wt and $m r p 14^{-/-}$mice at different time points after pneumococcal infection. Lung inflammation increased in time to a similar extent in wt and $m r p 14^{-/-}$ mice, revealing pathology consistent with severe pneumonia in both mouse strains (figure 4A-B).

Several studies have implicated MRP8/14 as a mediator of neutrophil recruitment. ${ }^{28-30}$ We determined myeloperoxidase (MPO) levels in whole-lung homogenates as a measure of neutrophil numbers (figure 4C). At $6 \mathrm{~h}$, lung MPO levels were low in both groups, but slightly higher in $m r p 14^{-/-}$mice $(\mathrm{p}<0.01$ vs wt mice); MPO concentrations were much higher at later time points and not different between groups.

As an additional readout for lung inflammation, we measured chemokines (MIP-2, KC) and cytokines (TNF- $\alpha$, IL-6, IL-10) in whole lung homogenates. Pulmonary cytokine and chemokine levels increased similarly in both mouse strains during the 

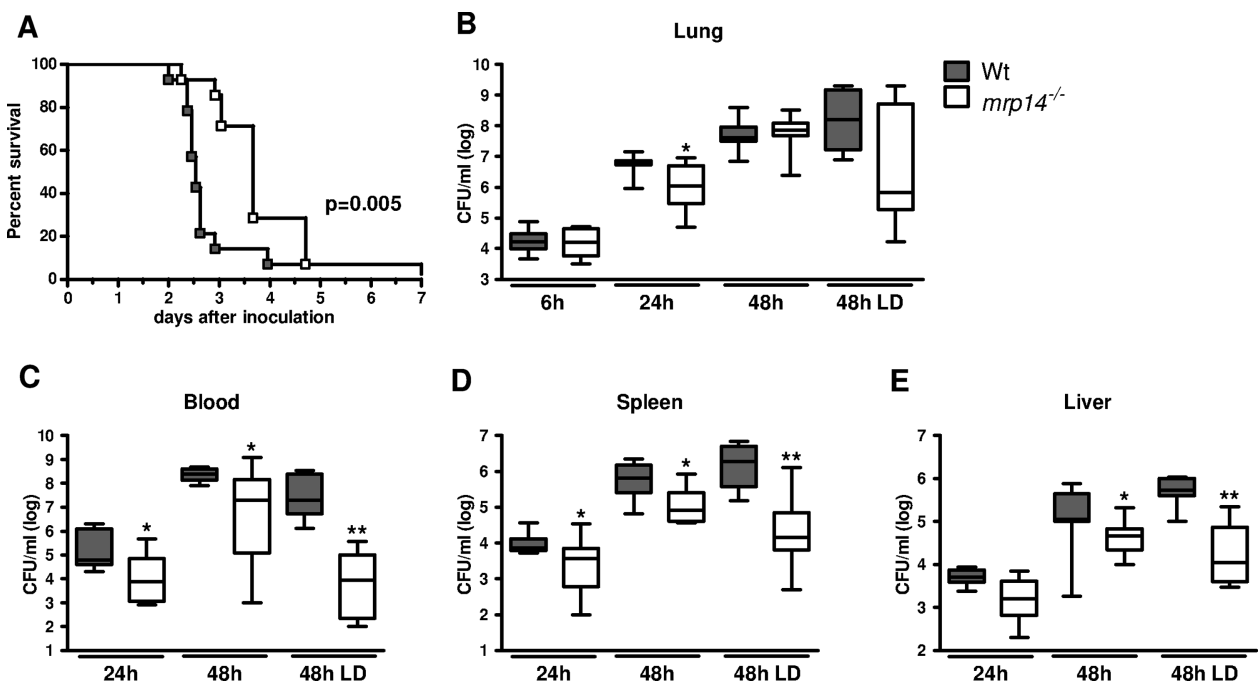

Figure $3 \mathrm{Mrp}^{-14^{-/}}$mice display diminished bacterial outgrowth and reduced mortality during pneumococcal pneumosepis. Survival of wildtype (wt) and $\mathrm{mrp}_{14}{ }^{-/-}$mice after intranasal infection with Streptococcus pneumoniae $\left(5 \times 10^{4} \mathrm{cfu}\right)$ ( $\mathrm{n}=15$ per group) (A). Bacterial loads in the lung (B), blood (C), spleen (D) and liver (E) after intranasal inoculation of $5 \times 10^{4} \mathrm{cfu}$ S. pneumoniae in wt (grey) and mrp14 ${ }^{-/-}$mice (white). In an additional experiment, we inoculated mice with $5 \times 10^{3} \mathrm{cfu}$ S. pneumoniae (indicated as LD=lower dose). Data are expressed as box-and-whisker diagrams depicting the smallest observation, lower quartile, median, upper quartile and largest observation (8 mice per group at each time point); ${ }^{*} p<0.05$, ${ }^{* *} p<0.01$ versus wt mice at the same time point.

course of the disease, showing no differences between $\operatorname{mrp} 14^{-/-}$ and wt mice (figure 4D-H). Also, $48 \mathrm{~h}$ after infection with $5 \times 10^{3}$ cfu $S$. pneumoniae, lung chemokine and cytokine levels did not differ between groups (data not shown). Altogether, these data indicate that MRP14 deficiency has little if any impact on the extent of lung inflammation induced by pneumococci.

\section{Impact of MRP14 deficiency on plasma cytokine levels}

We next determined the role of MRP8/14 in systemic inflammation. Mrp $14^{-/-}$mice showed lower plasma IL-6 concentrations at $48 \mathrm{~h}$ after infection with $5 \times 10^{4} \mathrm{cfu} S$. pneumoniae and lower plasma IL- 6 and TNF- $\alpha$ levels after administration of $5 \times 10^{3} \mathrm{cfu}$ pneumococci $(\mathrm{p}<0.01$ and 0.05 vs wt mice) (figure $5 \mathrm{~A}, \mathrm{~B})$. As these lower cytokine levels could be a consequence of lower bacterial loads in the blood of $m r p 14^{-/-}$mice, we harvested whole blood from wt and mrp14 $14^{-/-}$mice which was stimulated with increasing concentrations of growth-arrested $S$. pneumoniae. Wt and $m r p 14^{-/-}$whole blood produced similar TNF- $\alpha$ levels (figure 5C), suggesting that the lower systemic cytokine levels in mrp $14^{-/-}$mice might be a reflection of the lower bacterial numbers.

\section{MRP8/14 enhances pneumococcal growth by reducing} zinc toxicity

MRP8/14 can bind zinc and manganese, which results in growth inhibition of several micro-organisms. ${ }^{8-12} 3132$ While pneumococci also require zinc and manganese for growth, higher zinc concentrations competitively inhibit manganese uptake, rendering them hypersensitive to oxidative stress. ${ }^{14-17}$ Of interest, a previous study demonstrated an increase of serum zinc in murine pneumococcal pneumonia from approximately 15 to $640 \mu \mathrm{M}$. Manganese concentrations remained stable $(0.4-0.7 \mu \mathrm{M}) .{ }^{17}$ As MRP8/14 contains two metal-binding sites with high affinity for zinc, whereas, only one binds manganese, ${ }^{31} 32$ we hypothesised that metal binding by this heterodimer might be advantageous for the pathogen rather than for the host. We confirmed ${ }^{16} 17$ that increasing zinc concentrations (up to $200 \mu \mathrm{M}$ ) progressively inhibited pneumococcal growth in vitro in the presence of a constant manganese concentration $(0.1 \mu \mathrm{M})$ (figure $6 \mathrm{~A})$. By comparison, zinc did not influence the growth of Klebsiella pneumoniae in vitro under these conditions (figure 6B), suggesting that pneumococci indeed have a relative susceptibility towards a high zinc/manganese ratio. Importantly, recombinant mouse MRP8 or MRP8/14, but not MRP14, reduced $(100 \mu \mathrm{M})$ zinc-induced toxicity toward pneumococci (figure 6C). Without zinc addition, MRP8/14 $(100 \mu \mathrm{g} / \mathrm{mL})$ inhibited pneumococcal growth (figure 6D), mimicking its effect on other bacterial species. ${ }^{8-12}$ Together these data suggest that, in contrast with the antimicrobial effects exerted by the metal chelating properties of MRP8/14 on other bacterial species, the zinc-binding capacity of MRP8/14 is of benefit to $S$. pneumoniae.

\section{DISCUSSION}

Invasive infection is associated with the release of MRP8/14, which serves a complex role in a variety of innate defence mechanisms. MRP8/14 attracts neutrophils, ${ }^{28-30}$ mediates inflammatory processes via TLR4, ${ }^{7}$ and binds metals, which is antimicrobial against a range of bacterial ${ }^{8-10}$ and fungal ${ }^{11} 12$ pathogens. Here, we observed elevated MRP8/14 levels in the course of clinical and experimental Gram-positive pneumonia. In sharp contrast with earlier studies on the role of MRP8/14 in host defence against infection, ${ }^{8-12}$ we found that the presence of MRP8/14 was of benefit to $S$. pneumoniae rather than to the host, as reflected by reduced bacterial dissemination and delayed lethality of $m r p 14^{-/}$ mice in lethal pneumonia-derived sepsis. The mechanism by which S. pneumoniae exploits MRP8/14 likely involves the zinc chelating capacity of this heterodimer, thereby reducing zincmediated toxicity towards the pneumococcus.

We previously reported elevated MRP8/14 serum levels in patients with sepsis originating from different sources, including pneumonia. ${ }^{19}$ Accordingly, we demonstrate elevated serum MRP8/14 levels in newly admitted CAP patients. MRP8/14 levels were notably enhanced at the primary site of infection, as detected in BALF and lung tissue slides, corresponding to high levels in the abdominal cavity during peritoneal sepsis. ${ }^{19}$ 

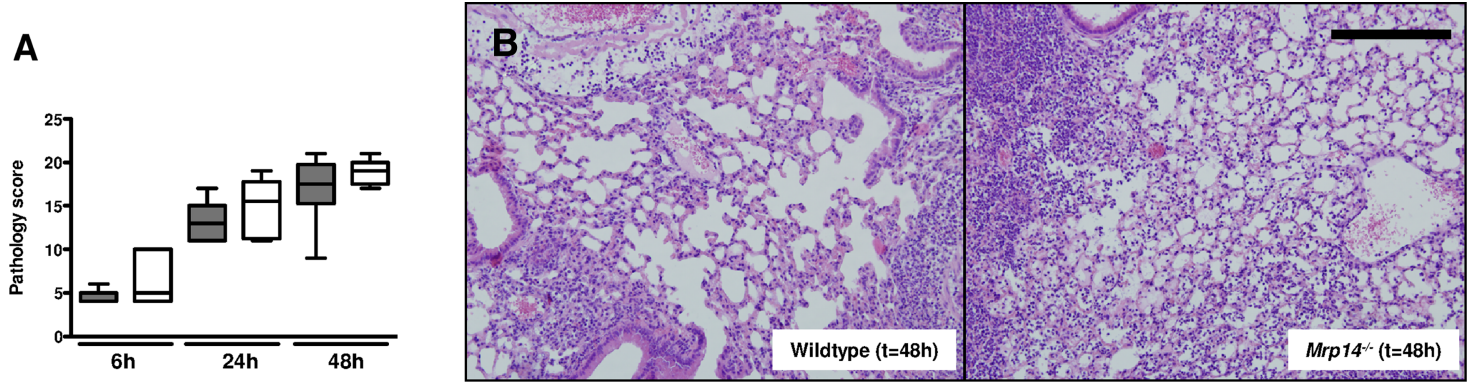

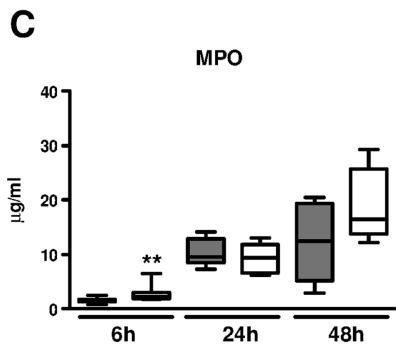

D

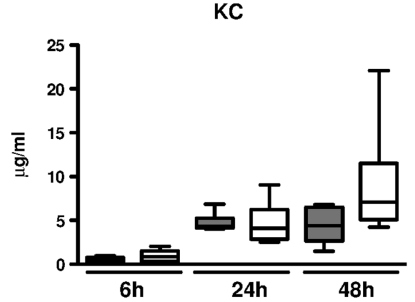

G

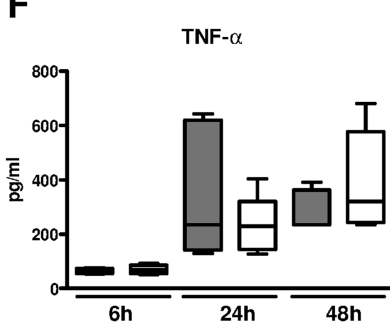

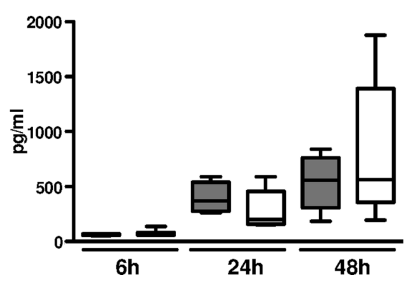

E

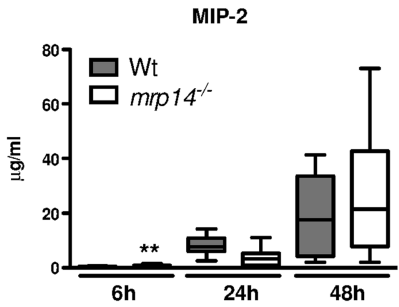

H

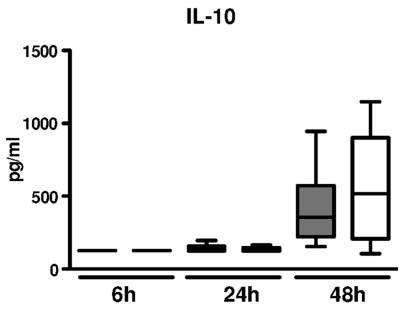

Figure 4 Pulmonary inflammation during pneumococcal pneumonia is not affected by loss of myeloid-related protein (MRP)8/14. Total pathology score at indicated time points after infection with Streptococcus pneumoniae $\left(5 \times 10^{4} \mathrm{cfu}\right.$ ) in wildtype (wt) (grey) and $\mathrm{mrp} 14^{-/-}$mice (white) determined according to the scoring system described in the online supplement (A). Data are expressed as box-and-whisker diagrams depicting the smallest observation, lower quartile, median, upper quartile and largest observation (8 mice per group at each time point). There were no statistically significant differences between the groups. Representative slides of lung H\&E stainings of wt and $m r p 14^{-/-}$mice $48 \mathrm{~h}$ after intranasal S. pneumoniae infection (B). Scale bar indicates $200 \mu \mathrm{m}$. Pulmonary MPO, indicative for the number of neutrophils (C), chemokine (KC and MIP-2) (D-E) and cytokine (TNF- $\alpha, \mathrm{IL}-6$ and IL-10) (F-H) 6, 24 and $48 \mathrm{~h}$ after intranasal S. pneumoniae infection in wt (grey) and mrp14 ${ }^{-/-}$mice (white). Data are expressed as box-and-whisker diagrams depicting the smallest observation, lower quartile, median, upper quartile and largest observation (8 mice per group at each time point); ${ }^{* *} p<0.01$ versus wt mice at the same time point.

Moreover, compartmentalised release of MRP8/14 was detected in healthy humans challenged with LTA in a lung subsegment. Together these data indicate that MRP8/14 is highly produced at the site of infection and released systemically during human pneumococcal pneumonia. Similarly, experimentally induced pneumococcal pneumonia resulted in high local and systemic release of MRP8/14 in mice, which is in agreement with earlier studies of murine bacterial pneumonia. ${ }^{9} 30$

Although MRP8/14 may exhibit proinflammatory effects under various pathophysiologic conditions, ${ }^{719}$ we were unable to demonstrate such a role during pneumonia. We observed largely unaltered pulmonary cytokine responses and lung
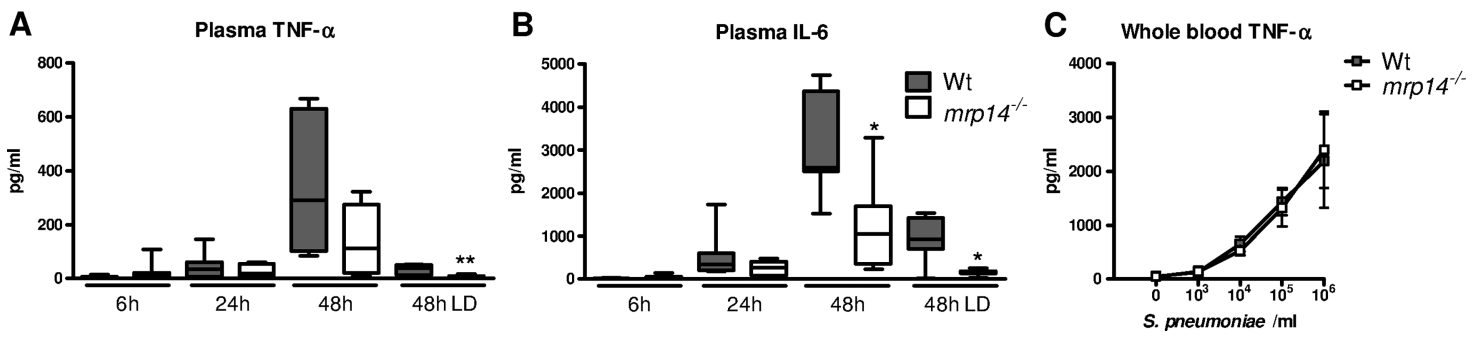

Figure 5 Plasma cytokine levels during pneumococcal pneumonia-derived sepsis. Plasma TNF- $\alpha$ (A) and IL-6 (B) 6,24 and $48 \mathrm{~h}$ after intranasal Streptococcus pneumoniae infection $\left(5 \times 10^{4} \mathrm{cfu}\right.$ ) in wildtype (wt) (grey) and $\mathrm{mrp} 14^{-/-}$mice (white). Data are expressed as box-and-whisker diagrams depicting the smallest observation, lower quartile, median, upper quartile and largest observation (8 mice per group at each time point). LD: lower dose $\left(5 \times 10^{3} \mathrm{cfu}\right) ;{ }^{*} \mathrm{p}<0.05,{ }^{* *} \mathrm{p}<0.01$ versus wt mice at the same time point. TNF- $\alpha$ levels after a $6 \mathrm{~h}$ stimulation of whole blood obtained from individual wt and $m r p 14^{-/-}$mice ( $n=4$ per group) with log-increasing concentrations of growth-arrested S. pneumoniae (C). Data are expressed as means \pm SEM (4 mice per group). 
A

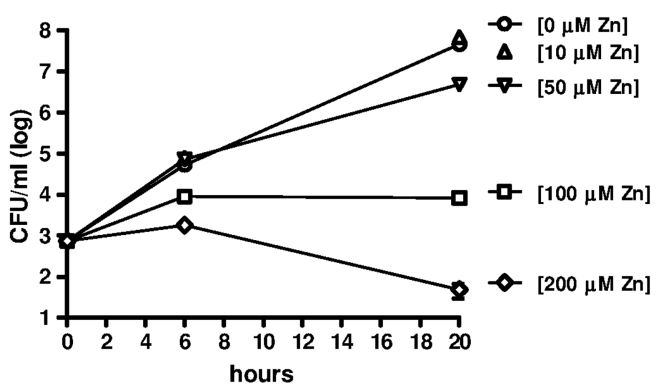

C

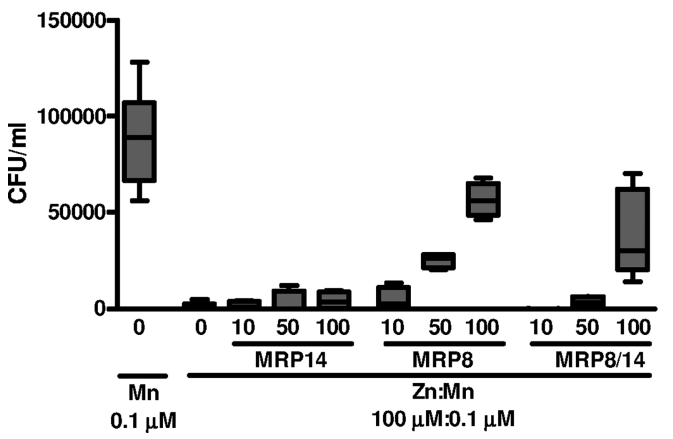

B

K. pneumoniae growth

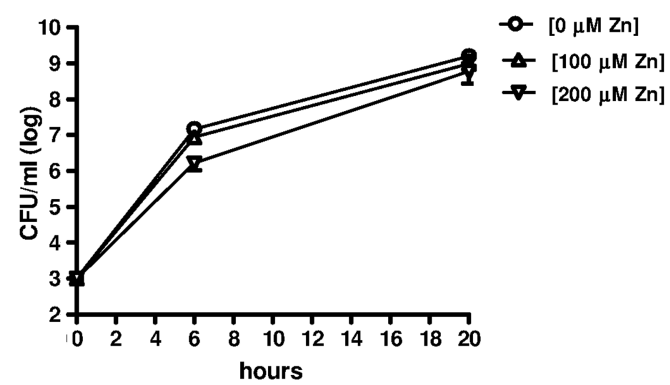

D

\section{S. pneumoniae growth}

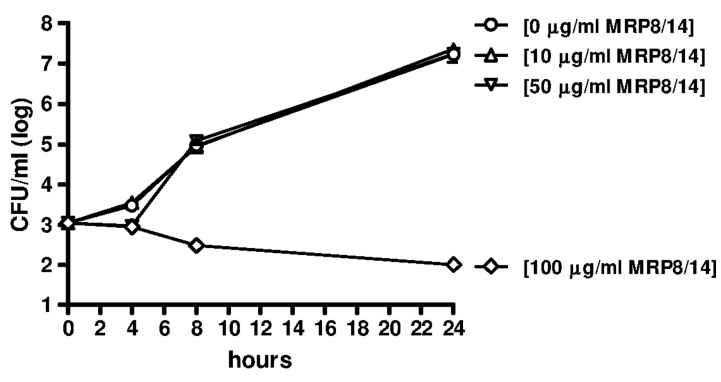

Figure 6 Myeloid-related protein (MRP)8/14 reduces zinc mediated toxicity towards pneumococci. Growth of Streptococcus pneumoniae (A) and Klebsiella pneumoniae (B) was assessed for 6 and $20 \mathrm{~h}$ in the presence of $0.1 \mu \mathrm{M}$ manganese and increasing concentrations of zinc. The growth-inhibiting effect of zinc towards pneumococci was reduced by increasing concentrations of MRP8 and MRP8/14 (C). MRP8/14 inhibits S. pneumoniae growth in zinc-free medium (D). Data are means \pm SEs of at least four replicates and representatives of triplicate experiments.

pathology. The reduced systemic cytokine levels in the current study were most likely caused by lower bacterial burdens in the circulation, as whole blood from $m r p 14^{-/}$mice produced similar TNF- $\alpha$ levels as wt blood upon stimulation with growth-arrested pneumococci. Previous studies have shown that MRP8/14 is able to potentiate lipopolysaccharide-induced signalling via TLR4, while by itself exerting only minimal proinflammatory effects. ${ }^{7}$ Although $S$. pneumoniae may activate TLR4 via its virulence factor pneumolysin, ${ }^{33}$ TLR2 is the predominant pattern recognition receptor that mediates lung inflammation induced by this pathogen. ${ }^{34}{ }^{35}$ Moreover, we previously reported that MRP8/14 does not contribute to lung inflammation during Gram-negative (Klebsiella) pneumonia ${ }^{9}$ wherein TLR4 is the main receptor driving the inflammatory response. $^{36} 37$ Together, these data indicate that endogenous MRP8/14 does not amplify inflammation in the lungs during pneumonia caused by a gradually growing bacterial load, that is, in the context of a likely clinical scenario. ${ }^{9}$

Many previous investigations have established an antibacterial role for MRP8/14 in in vivo infection models, ${ }^{8-12}$ which is dependent on the chelation of both zinc and manganese. ${ }^{31} 32$ Of note, MRP8 and MRP14 homodimers only bind zinc at their His-Glu-Xaa-Xaa-His (HEXXH) zinc-binding amino acid motif and do not impair microbial growth. ${ }^{38}$ When infected with pyogenic bacteria, such as S. aureus, ${ }^{8}$ K. pneumoniae ${ }^{9}$ or Acinetobacter baumanii, ${ }^{10}$ mrp $14^{-/-}$mice displayed enhanced bacterial outgrowth or mortality. In contrast to these earlier findings, lack of MRP8/14 reduced bacterial burdens in the lung, blood and distant organs and improved survival in pneumococcal pneumonia derived sepsis. This apparent inconsistent finding seemed surprising at first glance, especially since pneumococci, like the pathogens listed above, critically requires both zinc and manganese for normal (aerobic) growth. ${ }^{14-17}$ Higher zinc concentrations however, during pneumococcal infection, ${ }^{17}$ have significant toxicity towards this bacterium. ${ }^{16}{ }^{17}$ Zinc irreversibly binds to PsaA, thereby blocking intracellular uptake of manganese. Uptake of manganese is essential for manganese superoxide dismutase to render pneumococci less susceptible to oxidative stress and polymorphonuclear leukocyte killing. ${ }^{16} 17$ We postulate that high MRP8/14 concentrations reduce zinc/manganese ratios at infected body sites during pneumococcal pneumoniaderived sepsis, thereby providing a benefit for the pathogen rather than for the host. Previous investigations have indeed shown that MRP8/14 has two high-affinity metal binding sites for zinc, whereas only one is able to bind manganese. ${ }^{31} 32$ In our in vitro experiments, addition of recombinant MRP8/14 and MRP8 (most likely through zinc chelation at its HEXXH zincbinding site ${ }^{38}$ ), but not MRP14 partially restored growth of pneumococci in a high zinc/manganese ratio environment, revealing a likely mechanism for enhanced bacterial growth and, consequently, higher lethality in $\operatorname{mrp} 14^{-/-}$mice during pneumococcal sepsis. Such a mechanism would also explain the opposite phenotypes of $m r p 14^{-/}$mice during pneumonia caused by S. pneumoniae (reported here) and K. pneumoniae, ${ }^{9}$ since Klebsiella clearly proved not susceptible to high zinc concentrations. Of interest, very recently, Neisseria meningitidis was also reported to misuse MRP8/14 to promote its growth in vitro, albeit by a different mechanism, namely by binding MRP8/14 and using this protein as a zinc source in low zinc culture conditions. $^{39}$

A limitation of our study is that we were not able to directly measure zinc and manganese ratios at tissue level, at sites infected by pneumococci, as the state-of-the-art technique to measure localised metal distribution in tissues, that is, laser ablation inductively coupled plasma mass spectrometry, requires visible inflammatory lesions, ${ }^{8}$ which is not a pathologic feature 
of pneumococcal infection. Furthermore, to establish the role of MRP8/14 in the reduction of zinc toxicity and enhanced pneumococcal growth, additional studies should be conducted, such as supplementing mice with MRPs and/or feeding them with low or high zinc diets.

In conclusion, we document that human CAP is associated with profoundly elevated MRP8/14 concentrations in lungs and the circulation. Using our model of murine pneumococcal pneumonia, we show that the presence of high MRP8/14 levels is associated with enhanced bacterial outgrowth and lethality, most likely through binding of zinc by MRP8/14 and, thereby, reduction of zinc-mediated toxicity towards the bacterium. This study shows for the first time that the elemental binding properties of MRP8/14 can be misused by a clinical-relevant pathogen.

\section{Author affiliations}

${ }^{1}$ Center for Experimental and Molecular Medicine, University of Amsterdam, Amsterdam, The Netherlands

${ }^{2}$ Center for Infection and Immunity, Academic Medical Center, University of Amsterdam, Amsterdam, The Netherlands

${ }^{3}$ Institute of Immunology, University of Muenster, Muenster, Germany

${ }^{4}$ Intensive Care Department, Onze Lieve Vrouwe Gasthuis, Amsterdam, The Netherlands

${ }^{5}$ Department of Pathology, Microbiology and Immunology, Vanderbilt University School of Medicine, Nashville, Tennessee, USA

${ }^{6}$ Department of Critical Care Medicine, Saint-Luc University Hospital, Université Catholique de Louvain, Brussels, Belgium

${ }^{7}$ Department of Internal Medicine and Infectious Diseases, University Medical Center Utrecht, Utrecht, The Netherlands

${ }^{8}$ Department of Pathology, Academic Medical Center, University of Amsterdam, Amsterdam, The Netherlands

${ }^{9}$ Department of Intensive Care Medicine, University of Amsterdam, Amsterdam, The Netherlands

${ }^{10}$ Laboratory of Experimental Intensive Care and Anesthesiology, Academic Medical Center, University of Amsterdam, Amsterdam, The Netherlands

${ }^{11}$ Department of Pulmonology, St. Antonius Hospital, Nieuwegein, The Netherlands

${ }^{12}$ Division of Heart \& Lungs, University Medical Center Utrecht, Utrecht, The Netherlands

${ }^{13}$ Department of Internal Medicine, St. Antonius Hospital, Nieuwegein,

The Netherlands

${ }^{14}$ Division of Infectious Diseases, Academic Medical Center, University of

Amsterdam, The Netherlands

Acknowledgements The authors thank Marieke ten Brink and Joost Daalhuisen for their expert technical assistance during the animal experiments, and Regina de Beer for performing histopathological and immunohistochemical stainings.

Contributors AA performed the study, collected, analysed and interpreted the data and wrote the first draft of the article. TV, HE, BLM, MADvZ, YZ, JJH and SF assisted in collection and interpretation of the data. P-FL, XW, MJS, JCG and DHB provided human samples. JR, EPS, CvtV and AFdV assisted in interpretation of the data. TvdP designed the study and assisted in interpretation of the data. All authors contributed in revising the manuscript and finally approved the version of the manuscript to be published.

Funding This work is supported by a grant from the Landsteiner Foundation for Blood Transfusion Research (project LSBR 0706) and a clinical research talent fellowship of the University Medical Center Utrecht (2014 to MADvZ).

\section{Competing interests None.}

Ethics approval Scientific and ethics committees of the Academic Medical Center (Amsterdam, The Netherlands), St. Luke University Hospital (Brussels, Belgium), and/or St. Antonius Hospital (Nieuwegein, The Netherlands.

Provenance and peer review Not commissioned; externally peer reviewed.

\section{REFERENCES}

1 Mandell LA, Wunderink RG, Anzueto A, et al. Infectious Diseases Society of America/American Thoracic Society consensus guidelines on the management of community-acquired pneumonia in adults. Clin Infect Dis 2007:44(Suppl 2):S27-72.

2 Laterre $P F$, Garber $G$, Levy $\mathrm{H}$, et al. Severe community-acquired pneumonia as a cause of severe sepsis: data from the PROWESS study. Crit Care Med 2005:33:952-61.

3 van der Poll T, Opal SM. Pathogenesis, treatment, and prevention of pneumococcal pneumonia. Lancet 2009:374:1543-56.
4 Feldman C. Clinical relevance of antimicrobial resistance in the management of pneumococcal community-acquired pneumonia. J Lab Clin Med 2004:143:269-83.

5 Koppe U, Suttorp N, Opitz B. Recognition of Streptococcus pneumoniae by the innate immune system. Cell Microbiol 2012;14:460-6.

6 Leukert N, Vogl T, Strupat K, et al. Calcium-dependent tetramer formation of S100A8 and S100A9 is essential for biological activity. J Mol Biol 2006;359:961-72.

7 Vogl T, Tenbrock K, Ludwig S, et al. Mrp8 and Mrp14 are endogenous activators of Toll-like receptor 4, promoting lethal, endotoxin-induced shock. Nat Med 2007;13:1042-9.

8 Corbin BD, Seeley EH, Raab A, et al. Metal chelation and inhibition of bacterial growth in tissue abscesses. Science 2008;319:962-5.

9 Achouiti A, Vogl T, Urban CF, et al. Myeloid-related protein-14 contributes to protective immunity in gram-negative pneumonia derived sepsis. PLoS Pathog 2012:8:e1002987.

10 Hood Ml, Mortensen BL, Moore JL, et al. Identification of an Acinetobacter baumannii zinc acquisition system that facilitates resistance to calprotectin-mediated zinc sequestration. PLOS Pathog 2012;8:e1003068.

11 Bianchi M, Niemiec MJ, Siler U, et al. Restoration of anti-Aspergillus defense by neutrophil extracellular traps in human chronic granulomatous disease after gene therapy is calprotectin-dependent. J Allergy Clin Immunol 2011;127: $1243-52$

12 Urban CF, Ermert D, Schmid M, et al. Neutrophil extracellular traps contain calprotectin, a cytosolic protein complex involved in host defense against Candida albicans. PLoS Pathog 2009;5:e1000639.

13 Hood MI, Skaar EP. Nutritional immunity: transition metals at the pathogen-host interface. Nat Rev Microbiol 2012:10:525-37.

14 Berry AM, Paton JC. Sequence heterogeneity of PsaA, a 37-kilodalton putative adhesin essential for virulence of Streptococcus pneumoniae. Infect Immun 1996;64:5255-62.

15 Ogunniyi AD, Mahdi LK, Jennings MP, et al. Central role of manganese in regulation of stress responses, physiology, and metabolism in Streptococcus pneumoniae. J Bacteriol 2010;192:4489-97.

16 Jacobsen FE, Kazmierczak KM, Lisher JP, et al. Interplay between manganese and zinc homeostasis in the human pathogen Streptococcus pneumoniae. Metallomics 2011:3:38-41.

17 McDevitt $C A$, Ogunniyi AD, Valkov $E$, et al. A molecular mechanism for bacterial susceptibility to zinc. PLoS Pathog 2011;7:e1002357.

18 Bayle L, Chimalapati S, Schoehn G, et al. Zinc uptake by Streptococcus pneumoniae depends on both AdcA and AdcAll and is essential for normal bacterial morphology and virulence. Mol Microbiol 2011;82:904-16.

19 van Zoelen MA, Vogl T, Foell D, et al. Expression and role of myeloid-related protein-14 in clinical and experimental sepsis. Am J Respir Crit Care Med 2009:180:1098-106.

20 Wolthuis EK, Choi G, Dessing MC, et al. Mechanical ventilation with lower tidal volumes and positive end-expiratory pressure prevents pulmonary inflammation in patients without preexisting lung injury. Anesthesiology 2008;108:46-54.

21 Hoogerwerf JJ, de Vos AF, Bresser $\mathrm{P}$, et al. Lung inflammation induced by lipoteichoic acid or lipopolysaccharide in humans. Am J Respir Crit Care Med 2008;178:34-41

22 Morath S, Stadelmaier A, Geyer A, et al. Synthetic lipoteichoic acid from Staphylococcus aureus is a potent stimulus of cytokine release. J Exp Med 2002:195:1635-40.

23 Roth J, Burwinkel F, van den BC, et al. MRP8 and MRP14, S-100-like proteins associated with myeloid differentiation, are translocated to plasma membrane and intermediate filaments in a calcium-dependent manner. Blood 1993;82:1875-83.

24 Manitz MP, Horst B, Seeliger S, et al. Loss of S100A9 (MRP14) results in reduced interleukin-8-induced CD11b surface expression, a polarized microfilament system, and diminished responsiveness to chemoattractants in vitro. Mol Cell Biol 2003:23:1034-43.

25 Hobbs JA, May R, Tanousis K, et al. Myeloid cell function in MRP-14 (S100A9) null mice. Mol Cell Biol 2003;23:2564-76.

26 Rijneveld AW, Weijer S, Florquin S, et al. Thrombomodulin mutant mice with a strongly reduced capacity to generate activated protein $\mathrm{C}$ have an unaltered pulmonary immune response to respiratory pathogens and lipopolysaccharide. Blood 2004:103:1702-9.

27 Dessing MC, Knapp S, Florquin S, et al. CD14 facilitates invasive respiratory tract infection by Streptococcus pneumoniae. Am J Respir Crit Care Med 2007:175:604-11.

28 Viemann D, Strey A, Janning A, et al. Myeloid-related proteins 8 and 14 induce a specific inflammatory response in human microvascular endothelial cells. Blood 2005; 105:2955-62.

29 Vandal K, Rouleau P, Boivin A, et al. Blockade of S100A8 and S100A9 suppresses neutrophil migration in response to lipopolysaccharide. J Immuno/ 2003;171:2602-9.

30 Raquil MA, Anceriz N, Rouleau P, et al. Blockade of antimicrobial proteins S100A8 and $\mathrm{S100A9}$ inhibits phagocyte migration to the alveoli in streptococcal pneumonia. J Immunol 2008:180:3366-74. 
31 Damo SM, Kehl-Fie TE, Sugitani N, et al. Molecular basis for manganese sequestration by calprotectin and roles in the innate immune response to invading bacterial pathogens. Proc Natl Acad Sci USA 2013;110:3841-6.

32 Kehl-Fie TE, Chitayat S, Hood Ml, et al. Nutrient metal sequestration by calprotectin inhibits bacterial superoxide defense, enhancing neutrophil killing of Staphylococcus aureus. Cell Host Microbe 2011;10:158-64.

33 Malley R, Henneke P, Morse SC, et al. Recognition of pneumolysin by Toll-like receptor 4 confers resistance to pneumococcal infection. Proc Natl Acad Sci USA 2003;100:1966-71.

34 Schroder NW, Morath S, Alexander C, et al. Lipoteichoic acid (LTA) of Streptococcus pneumoniae and Staphylococcus aureus activates immune cells via Toll-like receptor (TLR)-2, lipopolysaccharide-binding protein (LBP), and CD14, whereas TLR-4 and MD-2 are not involved. J Biol Chem 2003;278:15587-94.
35 Knapp S, Wieland CW, van 't Veer $\mathrm{C}$, et al. Toll-like receptor 2 plays a role in the early inflammatory response to murine pneumococcal pneumonia but does not contribute to antibacterial defense. J Immunol 2004;172:3132-8.

36 Branger J, Knapp S, Weijer S, et al. Role of Toll-like receptor 4 in gram-positive and gram-negative pneumonia in mice. Infect Immun 2004;72:788-94.

37 Wieland CW, van Lieshout MH, Hoogendijk AJ, et al. Host defence during Klebsiella pneumonia relies on haematopoietic-expressed Toll-like receptors 4 and 2 . Eur Respir J 2011;37:848-57.

38 Sohnle PG, Hunter MJ, Hahn B, et al. Zinc-reversible antimicrobial activity of recombinant calprotectin (migration inhibitory factor-related proteins 8 and 14). J Infect Dis 2000;182:1272-5.

39 Stork M, Grijpstra J, Bos MP, et al. Zinc piracy as a mechanism of Neisseria meningitidis for evasion of nutritional immunity. PLoS Pathog 2013;9:e1003733. 